\title{
On some new general theorems for the explicit evaluations of Ramanujan's remarkable product of theta-functions
}

\author{
M.S. Mahadeva Naika $\cdot$ B.N. Dharmendra
}

Received: 22 December 2004 / Accepted: 12 August 2005 / Published online: 8 March 2008 (C) Springer Science+Business Media, LLC 2007

\begin{abstract}
On pages 338 and 339 in his first notebook (Notebooks (2 volumes), 1957), Ramanujan records eighteen values for a certain product of theta-functions. All these have been proved by B.C. Berndt, H.H. Chan and L.-C. Zhang (Proc. Edinburg Math. Soc. 40:583-612, 1997). The main purpose of this paper is to establish some new general theorems for the explicit evaluations of Ramanujan's product of theta-functions and some new explicit evaluations there from.
\end{abstract}

Keywords Class invariant $\cdot$ Modular equation $\cdot$ Theta-function

Mathematics Subject Classification (2000) 33D10 $\cdot 11 \mathrm{~F} 20 \cdot 11 \mathrm{~F} 27$

\section{Introduction}

In Chap. 16 of his second notebook [1, 4, 12], Ramanujan develops the theory of theta-functions and his theta-functions is defined by

$$
\begin{aligned}
& \varphi(q):=f(q, q)=\sum_{n=-\infty}^{\infty} q^{n^{2}}=\left(-q ; q^{2}\right)_{\infty}^{2}\left(q^{2} ; q^{2}\right)_{\infty} \\
& \psi(q):=f\left(q, q^{3}\right)=\sum_{n=0}^{\infty} q^{\frac{n(n+1)}{2}}=\frac{\left(q^{2} ; q^{2}\right)_{\infty}}{\left(q ; q^{2}\right)_{\infty}}
\end{aligned}
$$

M.S. Mahadeva Naika ( $\varangle) \cdot$ B.N. Dharmendra

Department of Mathematics, Bangalore University, Central College Campus, Bangalore, 560 001, India

e-mail: msmnaika@rediffmail.com

B.N. Dharmendra

e-mail: dharmamath@rediffmail.com 
and

$$
f(-q):=f\left(-q,-q^{2}\right)=\sum_{n=-\infty}^{\infty}(-1)^{n} q^{\frac{n(3 n-1)}{2}}=(q ; q)_{\infty}
$$

where

$$
(a ; q)_{\infty}=\prod_{n=0}^{\infty}\left(1-a q^{n}\right) .
$$

On page 338 in his first notebook [12, p. 338], Ramanujan defines

$$
a_{m, n}=\frac{n e^{\frac{-(n-1) \pi}{4} \sqrt{\frac{m}{n}}} \psi^{2}\left(e^{-\pi \sqrt{m n}}\right) \varphi^{2}\left(-e^{-2 \pi \sqrt{m n}}\right)}{\psi^{2}\left(e^{-\pi \sqrt{\frac{m}{n}}}\right) \varphi^{2}\left(-e^{-2 \pi \sqrt{\frac{m}{n}}}\right)} .
$$

He then, on pages 338 and 339, offers a list of eighteen particular values. All these eighteen values have been established by Berndt, Chan and Zhang [7].

Let $K, K^{\prime}, L$ and $L^{\prime}$ denote the complete elliptic integrals of the first kind associated with the moduli $k, k^{\prime}:=\sqrt{1-k^{2}}, l$ and $l^{\prime}:=\sqrt{1-l^{2}}$ respectively, where $0<k, l<1$. For a fixed positive integer $n$, suppose that

$$
n \frac{K^{\prime}}{K}=\frac{L^{\prime}}{L}
$$

Then a modular equation of degree $n$ is a relation between $k$ and $l$ induced by (1.5). Following Ramanujan, set $\alpha=k^{2}$ and $\beta=l^{2}$. Then we say $\beta$ is of degree $n$ over $\alpha$. Define

$$
\chi(q):=\left(-q ; q^{2}\right)_{\infty}
$$

and

$$
G_{n}:=2^{-\frac{1}{4}} q^{-\frac{1}{24}} \chi(q)
$$

where

$$
q=e^{-\pi \sqrt{r}}
$$

Moreover, if $q=e^{-\pi \sqrt{\frac{m}{n}}}$ and $\beta$ has degree $n$ over $\alpha$, then

$$
G_{\frac{m}{n}}=(4 \alpha(1-\alpha))^{\frac{-1}{24}}
$$

and

$$
G_{m n}=(4 \beta(1-\beta))^{\frac{-1}{24}} .
$$

The main purpose of this paper is to obtain several general theorems for the explicit evaluations of Ramanujan's product of theta-functions and also some new explicit evaluations as a consequence. 


\section{General theorems for the explicit evaluations of $a_{m, n}$}

In this section, we establish several new general formulas for the explicit evaluations of Ramanujan's remarkable product of theta-function.

Theorem 2.1 We have

$$
a_{m, n}=\frac{n e^{-\frac{(n-1) \pi}{4} \sqrt{\frac{m}{n}}} \psi^{2}\left(-e^{-\pi \sqrt{m n}}\right) \varphi^{2}\left(e^{-\pi \sqrt{m n}}\right)}{\psi^{2}\left(-e^{-\pi \sqrt{\frac{m}{n}}}\right) \varphi^{2}\left(e^{-\pi \sqrt{\frac{m}{n}}}\right)},
$$

where $m$ is any positive rational and $n$ is a positive integer.

Proof Using (1.1) and (1.2), we can easily find that

$$
\begin{aligned}
\psi(-q) \varphi\left(-q^{2}\right) & =\frac{\left(q^{2} ; q^{2}\right)_{\infty}}{\left(-q ; q^{2}\right)_{\infty}} \frac{\left(q^{2} ; q^{2}\right)_{\infty}}{\left(-q^{2} ; q^{2}\right)_{\infty}} \\
& =\frac{\left(q^{2} ; q^{2}\right)_{\infty}}{\left(q ; q^{2}\right)_{\infty}} \frac{\left(q ; q^{2}\right)_{\infty}\left(q^{2} ; q^{2}\right)_{\infty}}{\left(-q ; q^{2}\right)_{\infty}\left(-q^{2} ; q^{2}\right)_{\infty}} \\
& =\psi(q) \varphi(-q) .
\end{aligned}
$$

Replacing $q$ by $-q$ in the above identity, we obtain the required result with $q=e^{-\pi \sqrt{\frac{m}{n}}}$.

Theorem 2.2 If $m$ is any positive rational and $n$ is a positive integer, then

$$
a_{m, n} a_{\frac{1}{m}, n}=1
$$

Proof Using Entry 27 (iii) and (iv) of Chap. 16 of Ramanujan's notebooks [4, p. 43], we obtain

$$
\sqrt[4]{\alpha} e^{-\frac{\alpha}{8}} \psi\left(-e^{-\alpha}\right)=\sqrt[4]{\beta} e^{-\frac{\beta}{8}} \psi\left(-e^{-\beta}\right), \quad \alpha \beta=\pi^{2} .
$$

By Entry 27(i) of Chap. 16 of Ramanujan's notebooks [4, p. 43], we have

$$
\sqrt[4]{\alpha} \varphi\left(e^{-\alpha}\right)=\sqrt[4]{\beta} \varphi\left(e^{-\beta}\right), \quad \alpha \beta=\pi^{2} .
$$

Using (2.3) and (2.4) in (2.1), we obtain (2.2).

Corollary 2.1 We have

$$
a_{1, n}=1 \text {. }
$$

Proof Putting $m=1$ in (2.2), we obtain (2.5).

Remark A different proof of (2.3) can be found in [3]. 
Theorem 2.3 We have

$$
a_{m, n}=a_{n, m}
$$

Proof Using (2.3) and (2.4) in (2.1), we obtain (2.6).

Theorem 2.4 If $m$ is any positive rational,

$$
\begin{aligned}
& a_{m, 3}=\frac{3 e^{-\frac{\pi}{2} \sqrt{\frac{m}{3}}} \psi^{2}\left(-e^{-\pi \sqrt{3 m}}\right) \varphi^{2}\left(e^{-\pi \sqrt{3 m}}\right)}{\psi^{2}\left(-e^{-\pi \sqrt{\frac{m}{3}}}\right) \varphi^{2}\left(e^{-\pi \sqrt{\frac{m}{3}}}\right)}, \\
& P=\frac{\psi\left(-e^{-\pi \sqrt{\frac{m}{3}}}\right)}{e^{-\frac{\pi}{4} \sqrt{\frac{m}{3}}} \psi\left(-e^{-\pi \sqrt{3 m}}\right)} \quad \text { and } \quad Q=\frac{\varphi\left(e^{-\pi \sqrt{\frac{m}{3}}}\right)}{\varphi\left(e^{-\pi \sqrt{3 m}}\right)},
\end{aligned}
$$

then

$$
a_{m, 3}^{2}=\frac{9\left(1+P^{4}\right)}{P^{4}\left(9+P^{4}\right)}=\frac{9\left(1-Q^{4}\right)}{Q^{4}\left(Q^{4}-9\right)}, \quad Q^{4} \neq 9 .
$$

Proof By Theorems 9.9 and 9.10 of Chap. 33 of Ramanujan notebooks [6, p. 148], we find that

$$
b(q):=\varphi(-q)=(1-x)^{\frac{1}{8}} \sqrt{z}
$$

and

$$
c(q):=2^{\frac{3}{4}} q^{\frac{1}{8}} \psi(q)=x^{\frac{1}{8}} \sqrt{z} .
$$

Using (2.10) and (2.11) in Theorem 10.5 of Chap. 33 of Ramanujan's notebooks $[6$, p. 156], we find that

$$
\frac{\psi^{4}(-q)}{q \psi^{4}\left(-q^{3}\right)}+9=\frac{\varphi^{4}(q)}{\varphi^{4}\left(q^{3}\right)}+\frac{\psi^{4}(-q)}{q \psi^{4}\left(-q^{3}\right)} \frac{\varphi^{4}(q)}{\varphi^{4}\left(q^{3}\right)} .
$$

Putting $n=3$ and $q=e^{-\pi \sqrt{\frac{m}{3}}}$ in (2.1), we find that

$$
a_{m, 3}=\frac{3 q^{\frac{1}{2}} \psi^{2}\left(-q^{3}\right) \varphi^{2}\left(q^{3}\right)}{\psi^{2}(-q) \varphi^{2}(q)} .
$$

Using (2.13) in (2.12), we obtain (2.9).

Corollary 2.2 We have

$$
a_{3,3}=\frac{1}{\sqrt{3}}
$$

Proof Putting $m=3$ in (2.8) and from the Ramanujan's notebooks [6, p. 327], we have

$$
Q=\frac{\varphi\left(e^{-\pi}\right)}{\varphi\left(e^{-3 \pi}\right)}=\sqrt[4]{6 \sqrt{3}-9}
$$


Using (2.15) in (2.9), we obtain the required result.

Theorem 2.5 If $m$ is any positive rational,

$$
\begin{aligned}
& a_{m, 5}=\frac{5 e^{-\pi \sqrt{\frac{m}{5}}} \psi^{2}\left(-e^{-\pi \sqrt{5 m}}\right) \varphi^{2}\left(e^{-\pi \sqrt{5 m}}\right)}{\psi^{2}\left(-e^{-\pi \sqrt{\frac{m}{5}}}\right) \varphi^{2}\left(e^{-\pi \sqrt{\frac{m}{5}}}\right)}, \\
& P=\frac{\psi\left(-e^{-\pi \sqrt{\frac{m}{5}}}\right)}{e^{-\pi \sqrt{\frac{m}{5}}} \psi\left(-e^{-\pi \sqrt{5 m}}\right)} \quad \text { and } \quad Q=\frac{\varphi\left(e^{-\pi \sqrt{\frac{m}{5}}}\right)}{\varphi\left(e^{-\pi \sqrt{5 m}}\right)},
\end{aligned}
$$

then

$$
a_{m, 5}=\frac{5\left(1+P^{2}\right)}{P^{2}\left(5+P^{2}\right)}=\frac{5\left(1-Q^{2}\right)}{Q^{2}\left(Q^{2}-5\right)}, \quad Q \neq \sqrt{5}
$$

Proof Using (2.10) and (2.11) in Theorem 10.6 of Chap. 33 of Ramanujan's notebooks [6, p. 157], we find that

$$
\frac{\psi^{2}(-q)}{q \psi^{2}\left(-q^{5}\right)}+5=\frac{\varphi^{2}(q)}{\varphi^{2}\left(q^{5}\right)}+\frac{\psi^{2}(-q)}{q \psi^{2}\left(-q^{5}\right)} \frac{\varphi^{2}(q)}{\varphi^{2}\left(q^{5}\right)} .
$$

Putting $n=5$ and $q=e^{-\pi \sqrt{\frac{m}{5}}}$ in (2.1), we find that

$$
a_{m, 5}=\frac{5 q \psi^{2}\left(-q^{5}\right) \varphi^{2}\left(q^{5}\right)}{\psi^{2}(-q) \varphi^{2}(q)} .
$$

Using (2.20) in (2.19), we obtain the required result.

Corollary 2.3 We have

$$
a_{5,5}=\frac{1}{5}
$$

Proof Putting $m=5$ in (2.17) and from the Ramanujan's notebooks [6, p. 327], we have

$$
\frac{\varphi\left(e^{-\pi}\right)}{\varphi\left(e^{-5 \pi}\right)}=\sqrt{5 \sqrt{5}-10} .
$$

Using (2.21) in (2.18), we obtain the required result.

Theorem 2.6 If $m$ is any positive rational,

$$
\begin{aligned}
& a_{m, 9}=\frac{9 e^{-2 \pi \sqrt{\frac{m}{9}}} \psi^{2}\left(-e^{-\pi \sqrt{9 m}}\right) \varphi^{2}\left(e^{-\pi \sqrt{9 m}}\right)}{\psi^{2}\left(-e^{-\pi \sqrt{\frac{m}{9}}}\right) \varphi^{2}\left(e^{-\pi \sqrt{\frac{m}{9}}}\right)}, \\
& P=\frac{\psi\left(-e^{-\pi \sqrt{\frac{m}{9}}}\right)}{e^{-\pi \sqrt{\frac{m}{9}}} \psi\left(-e^{-\pi \sqrt{9 m}}\right)} \quad \text { and } \quad Q=\frac{\varphi\left(e^{-\pi \sqrt{\frac{m}{9}}}\right)}{\varphi\left(e^{-\pi \sqrt{9 m}}\right)},
\end{aligned}
$$


then

$$
a_{m, 9}=\frac{9(1+P)^{2}}{P^{2}(3+P)^{2}}=\frac{9(1-Q)^{2}}{Q^{2}(3-Q)^{2}}, \quad Q \neq 3 .
$$

Proof Using (2.10) and (2.11) in Theorem 10.7 of Chap. 33 of Ramanujan's notebooks $[6$, p. 158], we find that

$$
\frac{\psi(-q)}{q \psi\left(-q^{9}\right)}+3=\frac{\varphi(q)}{\varphi\left(q^{9}\right)}+\frac{\psi(-q)}{q \psi\left(-q^{9}\right)} \frac{\varphi(q)}{\varphi\left(q^{9}\right)} .
$$

Putting $n=9$ and $q=e^{-\pi \sqrt{\frac{m}{9}}}$ in (2.1), we find that

$$
a_{m, 9}=\frac{9 q \psi^{2}\left(-q^{9}\right) \varphi^{2}\left(q^{9}\right)}{\psi^{2}(-q) \varphi^{2}(q)} .
$$

Using (2.26) in (2.25), we obtain the required result.

Corollary 2.4 We have

$$
a_{9,9}=\frac{(1+\sqrt[3]{2(\sqrt{3}+1)})^{2}(\sqrt[3]{2(\sqrt{3}+1)}-2)^{2}}{9(\sqrt[3]{2(\sqrt{3}+1)})^{2}} .
$$

Proof Putting $m=9$ in (2.23) and from Ramanujan's notebooks [6, p. 328], we have

$$
Q=\frac{\varphi\left(e^{-\pi}\right)}{\varphi\left(e^{-9 \pi}\right)}=\frac{3}{1+\sqrt[3]{2(\sqrt{3}+1)}} .
$$

Using (2.28) in (2.24), we obtain (2.27).

Remark Ramanujan recorded many values of $\frac{\varphi\left(e^{-\pi}\right)}{\varphi\left(e^{-n \pi}\right)}$ for $n=3,5,7,9$ and 45 in his notebooks [6, p. 327-328]. One can easily obtain the values of $a_{m, n}$ by using these values in Theorems 2.4, 2.5 and 2.6.

Theorem 2.7 If $m$ is any positive rational,

$$
a_{m, 9}=\frac{9 e^{-2 \pi \sqrt{\frac{m}{9}}} \psi^{2}\left(-e^{-\pi \sqrt{9 m}}\right) \varphi^{2}\left(e^{-\pi \sqrt{9 m}}\right)}{\psi^{2}\left(-e^{-\pi \sqrt{\frac{m}{9}}}\right) \varphi^{2}\left(e^{-\pi \sqrt{\frac{m}{9}}}\right)}
$$

and

$$
V_{m}=\frac{G_{\frac{m}{9}}^{3}}{G_{9 m}^{3}}
$$

then

$$
\sqrt{a_{m, 9}}+\frac{1}{\sqrt{a_{m, 9}}}=\frac{1}{3}\left(V_{m}+\frac{1}{V_{m}}+4\right) .
$$


Proof By Entry 56 of Chap. 25 of Ramanujan's notebooks [5, (56.7), p. 211], we have

$$
R^{2} Q^{2}-3 R Q=Q^{3}-R^{3}
$$

where

$$
R=\frac{f(q)}{q^{\frac{1}{3}} f\left(q^{9}\right)}
$$

and

$$
Q=\frac{f\left(-q^{2}\right)}{q^{\frac{2}{3}} f\left(-q^{18}\right)} .
$$

Squaring both sides of (2.32), we obtain

$$
R Q+\frac{9}{R Q}=\left(\frac{Q}{R}\right)^{3}+\left(\frac{R}{Q}\right)^{3}+4
$$

Let $q=e^{-\pi \sqrt{\frac{m}{9}}}$, then the identity (2.29), becomes

$$
a_{m, 9}=\frac{9 q^{2} \psi^{2}\left(-q^{9}\right) \varphi^{2}\left(q^{9}\right)}{\psi^{2}(-q) \varphi^{2}(q)} .
$$

Using Entry 24(iii) of Chap. 16 of Ramanujan's notebooks [4, p. 39] in (2.34), we obtain

$$
a_{m, 9}=\frac{9 q^{2} f^{2}\left(q^{9}\right) f^{2}\left(-q^{18}\right)}{f^{2}(q) f^{2}\left(-q^{2}\right)} .
$$

Using (2.35) in (2.33), we find that

$$
\frac{3}{\sqrt{a_{m, 9}}}+3 \sqrt{a_{m, 9}}=\frac{G_{\frac{m}{9}}^{3}}{G_{9 m}^{3}}+\frac{G_{9 m}^{3}}{G_{\frac{m}{9}}^{3}}+4 .
$$

Using (2.30) in (2.36) and after some simplification, we obtain (2.31).

Corollary 2.5 We have

$$
\begin{aligned}
& \text { (i) } a_{2,9}=(2+\sqrt{3})(5-2 \sqrt{6}), \\
& \text { (ii) } a_{25,9}=(16+4 \sqrt{15}-\sqrt{495+128 \sqrt{15}})^{2} .
\end{aligned}
$$

Proof of (2.37) Putting $m=2$ in (2.31), we obtain

$$
\sqrt{a_{2,9}}+\frac{1}{\sqrt{a_{2,9}}}=\frac{1}{3}\left(V_{2}+V_{2}^{-1}+4\right),
$$


where

$$
V_{2}=\frac{G_{\frac{2}{9}}^{3}}{G_{18}^{3}} .
$$

By Theorem 4.2 (v) and (vi) in [2] and [11], we have

$$
G_{\frac{2}{9}}=2^{-\frac{1}{8}}(\sqrt{3}-\sqrt{2})^{\frac{1}{12}}\left((\sqrt{3}-\sqrt{2})^{2}+7 \sqrt{2}\right)^{\frac{1}{8}}
$$

and

$$
G_{18}=2^{-\frac{1}{8}}(\sqrt{3}+\sqrt{2})^{\frac{1}{12}}\left((\sqrt{3}+\sqrt{2})^{2}+7 \sqrt{2}\right)^{\frac{1}{8}} .
$$

Using (2.40) and (2.41) in (2.39) and then solving the quadratic equation, we obtain (2.37).

Proof of (2.38) Putting $m=25$ in (2.31), we obtain

$$
\sqrt{a_{25,9}}+\frac{1}{\sqrt{a_{25,9}}}=\frac{1}{3}\left(V_{25}+V_{25}^{-1}+4\right),
$$

where

$$
V_{25}=\frac{G_{\frac{25}{9}}^{3}}{G_{225}^{3}} .
$$

From the table of Chap. 34 of Ramanujan's notebooks [6, p. 195], we have

$$
G_{225}=(2+\sqrt{3})^{\frac{1}{3}}\left(\frac{\sqrt{5}+1}{4}\right)(\sqrt{4+\sqrt{15}}+\sqrt[4]{15}) .
$$

From page 149 of Chap. 4 in [10, 13(c), p. 147], we have

$$
G_{\frac{25}{9}}=(2+\sqrt{3})^{\frac{1}{3}}\left(\frac{\sqrt{5}+1}{4}\right)(\sqrt{4+\sqrt{15}}-\sqrt[4]{15}) .
$$

Using (2.43) and (2.44) in (2.42) and then solving the quadratic equation, we obtain (2.38).

Theorem 2.8 If $m$ is any positive rational,

$$
a_{m, 13}=\frac{13 e^{-3 \pi \sqrt{\frac{m}{13}}} \psi^{2}\left(-e^{-\pi \sqrt{13 m}}\right) \varphi^{2}\left(e^{-\pi \sqrt{13 m}}\right)}{\psi^{2}\left(-e^{-\pi \sqrt{\frac{m}{13}}}\right) \varphi^{2}\left(e^{-\pi \sqrt{\frac{m}{13}}}\right)}
$$

and

$$
V_{m}=\frac{G_{\frac{m}{13}}}{G_{13 m}}
$$


then

$$
\sqrt{a_{m, 13}}-\frac{1}{\sqrt{a_{m, 13}}}=\frac{1}{\sqrt{13}}\left(V_{m}-\frac{1}{V_{m}}\right)\left(\left(V_{m}-\frac{1}{V_{m}}\right)^{2}+7\right) .
$$

Proof By Entry 57 of Chap. 25 of Ramanujan's notebooks [5, (57.6), p. 212], we have

$$
R Q-\frac{13}{R Q}=\left(\frac{Q}{R}\right)^{3}-\left(\frac{R}{Q}\right)^{3}+4\left(\frac{Q}{R}\right)-4\left(\frac{R}{Q}\right)
$$

where

$$
R=\frac{f(q)}{q^{\frac{1}{2}} f\left(q^{13}\right)}
$$

and

$$
Q=\frac{f\left(-q^{2}\right)}{q f\left(-q^{26}\right)} .
$$

Using Entry 24(iii) of Chap. 16 of Ramanujan's notebooks [4, p. 39] in (2.45), we find that

$$
a_{m, 13}=\frac{13 q^{3} f^{2}\left(q^{13}\right) f^{2}\left(-q^{26}\right)}{f^{2}(q) f^{2}\left(-q^{2}\right)}
$$

where $q=e^{-\pi \sqrt{\frac{m}{13}}}, m$ is any positive rational. Using (2.49) in (2.48), we deduce that

$$
\sqrt{\frac{13}{a_{m, 13}}}-\sqrt{13 a_{m, 13}}=\frac{G_{13 m}^{3}}{G_{\frac{m}{13}}^{3}}-\frac{G_{\frac{m}{13}}^{3}}{G_{13 m}^{3}}+4 \frac{G_{13 m}}{G_{\frac{m}{13}}}-4 \frac{G_{\frac{m}{13}}}{G_{13 m}} .
$$

Using (2.46) in (2.50) and after some simplification of the above identity (2.50), we obtain (2.47).

\section{Corollary 2.6 We have}

$$
\begin{aligned}
& \text { (i) } a_{9,13}=\frac{(-\sqrt{3+4 \sqrt{3}}(5+2 \sqrt{3})+\sqrt{13}(4+2 \sqrt{3}))^{2}}{13} \text {, } \\
& \text { (ii) } a_{21,13}=(8-3 \sqrt{7})^{2}(\sqrt{13}-2 \sqrt{3})^{2} .
\end{aligned}
$$

Proof of (2.51) Putting $m=9$ in (2.47), we find that

$$
\sqrt{a_{9,13}}-\frac{1}{\sqrt{a_{9,13}}}=\frac{1}{\sqrt{13}}\left(V_{9}-V_{9}^{-1}\right)\left(\left(V_{9}-V_{9}^{-1}\right)^{2}+7\right),
$$

where

$$
V_{9}=\frac{G_{\frac{9}{13}}}{G_{117}} .
$$


By Entry 41 of Chap. 36 of Ramanujan's notebooks [6, p. 378] with $P=\frac{1}{G_{13 n} G_{\frac{n}{13}}}$ and $Q=\frac{G_{13 n}}{G_{\frac{n}{13}}}$, we find that

$$
\begin{aligned}
& \left(\frac{G_{13 n}}{G_{\frac{n}{13}}}\right)^{7}+\left(\frac{G_{\frac{n}{13}}}{G_{13 n}}\right)^{7} \\
& +13\left(\left(\frac{G_{13 n}}{G_{\frac{n}{13}}}\right)^{5}+\left(\frac{G_{\frac{n}{13}}}{G_{13 n}}\right)^{5}+52\left(\left(\frac{G_{13 n}}{G_{\frac{n}{13}}}\right)^{3}+\left(\frac{G_{\frac{n}{13}}}{G_{13 n}}\right)^{3}\right)\right) \\
& +78\left(\left(\frac{G_{13 n}}{G_{\frac{n}{13}}}\right)+\left(\frac{G_{\frac{n}{13}}}{G_{13 n}}\right)\right)-8\left(\frac{1}{G_{\frac{n}{13}}^{6} G_{13 n}^{6}}-G_{\frac{n}{13}}^{6} G_{13 n}^{6}\right)=0
\end{aligned}
$$

From the table of Chap. 34 of Ramanujan's notebooks [6, p. 193], we have

$$
G_{117}=(2 \sqrt{3}+\sqrt{13})^{\frac{1}{6}}\left(\frac{3+\sqrt{13}}{2}\right)\left(\frac{\sqrt{4+\sqrt{13}}+\sqrt[4]{3}}{2}\right) .
$$

Using (2.55) in (2.54) with $n=9$, we find that

$$
G_{\frac{9}{13}}=(2 \sqrt{3}+\sqrt{13})^{\frac{1}{6}}\left(\frac{3+\sqrt{13}}{2}\right)\left(\frac{\sqrt{4+\sqrt{13}}-\sqrt[4]{3}}{2}\right) .
$$

Using (2.55) and (2.56) in (2.53) and then solving the quadratic equation, we obtain (2.51).

Proof of (2.52) Putting $m=21$ in (2.47), we find that

$$
\sqrt{a_{21,13}}-\frac{1}{\sqrt{a_{21,13}}}=\frac{1}{\sqrt{13}}\left(V_{21}-V_{21}^{-1}\right)\left(\left(V_{21}-V_{21}^{-1}\right)^{2}+7\right),
$$

where

$$
V_{21}=\frac{G_{\frac{21}{13}}}{G_{273}} .
$$

From the table of Chap. 34 of Ramanujan's notebooks [6, p. 196], we have

$$
G_{273}=(2+\sqrt{3})^{\frac{1}{4}}\left(\frac{3+\sqrt{13}}{2}\right)^{\frac{1}{2}}\left(\frac{\sqrt{7}+\sqrt{3}}{2}\right)^{\frac{1}{2}}\left(\frac{15 \sqrt{7}+11 \sqrt{13}}{2}\right)^{\frac{1}{6}}
$$

Using (2.58) in (2.54) with $n=21$, we find that

$$
G_{\frac{21}{13}}=(2+\sqrt{3})^{\frac{1}{4}}\left(\frac{\sqrt{13}-3}{2}\right)^{\frac{1}{2}}\left(\frac{\sqrt{7}-\sqrt{3}}{2}\right)^{\frac{1}{2}}\left(\frac{15 \sqrt{7}+11 \sqrt{13}}{2}\right)^{\frac{1}{6}}
$$


Using (2.59) and (2.58) in (2.57) and then solving the quadratic equation, we obtain (2.52).

Theorem 2.9 If $m$ is any positive rational,

$$
\begin{aligned}
& a_{m, 25}=\frac{25 e^{-6 \pi \sqrt{\frac{m}{25}}} \psi^{2}\left(-e^{-\pi \sqrt{25 m}}\right) \varphi^{2}\left(e^{-\pi \sqrt{25 m}}\right)}{\psi^{2}\left(-e^{-\pi \sqrt{\frac{m}{25}}}\right) \varphi^{2}\left(e^{-\pi \sqrt{\frac{m}{25}}}\right)}, \\
& V_{m}=\frac{G_{\frac{m}{25}}}{G_{25 m}},
\end{aligned}
$$

then

$$
\sqrt{a_{m, 25}}+\frac{1}{\sqrt{a_{m, 25}}}=\frac{1}{5}\left[\left(V_{m}+V_{m}^{-1}\right)\left[\left(V_{m}+V_{m}^{-1}+2\right)^{2}-7\right]-8\right] .
$$

Proof By Entry 58 of Chap. 25 of Ramanujan's notebooks [5, (58.7), p. 213], we have

$$
R Q+\frac{25}{R Q}=\left(\frac{Q}{R}\right)^{3}+\left(\frac{R}{Q}\right)^{3}+4\left(\frac{Q}{R}\right)^{2}+4\left(\frac{R}{Q}\right)^{2}
$$

where

$$
R=\frac{f(q)}{q f\left(q^{25}\right)}
$$

and

$$
Q=\frac{f\left(-q^{2}\right)}{q^{2} f\left(-q^{50}\right)} .
$$

Using Entry 24(iii) of Chap. 16 of Ramanujan's notebooks [4, p. 39] with $q=$ $e^{-\pi \sqrt{\frac{m}{25}}}$, we obtain

$$
a_{m, 25}=\frac{25 q^{6} f^{2}\left(q^{25}\right) f^{2}\left(-q^{50}\right)}{f^{2}(q) f^{2}\left(-q^{2}\right)} .
$$

Using (2.64) in (2.63) and then using (2.61) in the resultant identity, we obtain the required result (2.62).

\section{General formulas for explicit evaluations of $a_{m, p} a_{q^{2} m, p}$}

In this section, we establish several new general formulas for the explicit evaluations of Ramanujan's remarkable product of theta-functions involving $a_{m, p}$ and $a_{q^{2} m, p}$.

Theorem 3.1 If $m$ is any positive rational,

$$
A_{m}=a_{m, 3} a_{25 m, 3}
$$




$$
\begin{aligned}
= & \frac{9 e^{-\pi \sqrt{3 m}} \psi^{2}\left(-e^{-\pi \sqrt{3 m}}\right) \varphi^{2}\left(e^{-\pi \sqrt{3 m}}\right) \psi^{2}\left(-e^{-5 \pi \sqrt{3 m}}\right) \varphi^{2}\left(e^{-5 \pi \sqrt{3 m}}\right)}{\psi^{2}\left(-e^{-\pi \sqrt{\frac{m}{3}}}\right) \varphi^{2}\left(e^{-\pi \sqrt{\frac{m}{3}}}\right) \psi^{2}\left(-e^{-5 \pi \sqrt{\frac{m}{3}}}\right) \varphi^{2}\left(e^{-5 \pi \sqrt{\frac{m}{3}}}\right)}, \\
V_{m} & =\frac{G_{\frac{m}{3}} G_{\frac{25 m}{3}}}{G_{3 m} G_{75 m}}
\end{aligned}
$$

then

$$
\sqrt{A_{m}}-\frac{1}{\sqrt{A_{m}}}=\frac{1}{3}\left(V_{m}-V_{m}^{-1}\right)\left[\left(V_{m}-V_{m}^{-1}\right)^{2}+7\right] .
$$

Proof By Entry 60 of Chap. 25 of Ramanujan's notebooks [5, (60.5), p. 216], we have

$$
R Q-\frac{9}{R Q}=\left(\frac{Q}{R}\right)^{3}-\left(\frac{R}{Q}\right)^{3}+4\left(\frac{Q}{R}\right)-4\left(\frac{R}{Q}\right)
$$

where

$$
R=\frac{f(q) f\left(q^{5}\right)}{q^{\frac{1}{2}} f\left(q^{3}\right) f\left(q^{15}\right)}
$$

and

$$
Q=\frac{f\left(-q^{2}\right) f\left(-q^{10}\right)}{q f\left(-q^{6}\right) f\left(-q^{30}\right)} .
$$

Using Entry 24(iii) of Chap. 16 of Ramanujan's notebooks [4, p. 39] with $q=$ $e^{-\pi \sqrt{\frac{m}{3}}}$ in (3.1), we obtain

$$
A_{m}=a_{m, 3} a_{25 m, 3}=\frac{9 q^{3} f^{2}\left(q^{3}\right) f^{2}\left(q^{15}\right) f^{2}\left(-q^{6}\right) f\left(-q^{30}\right)}{f^{2}(q) f^{2}\left(q^{5}\right) f^{2}\left(-q^{2}\right) f\left(-q^{10}\right)} .
$$

Using (3.2) and (3.5) in (3.4), we find that

$$
\frac{3}{\sqrt{A_{m}}}-3 \sqrt{A_{m}}=V_{m}^{-3}-V_{m}^{3}+4 V_{m}^{-1}-4 V_{m} .
$$

On simplification of the above identity (3.6), we obtain the required result (3.3).

Theorem 3.2 If $m$ is any positive rational,

$$
\begin{aligned}
A_{m} & :=\frac{a_{m, 3}}{a_{25 m, 3}} \\
& =\frac{e^{2 \pi \sqrt{\frac{m}{3}}} \psi^{2}\left(-e^{-\pi \sqrt{3 m}}\right) \varphi^{2}\left(e^{-\pi \sqrt{3 m}}\right) \psi^{2}\left(-e^{-5 \pi \sqrt{\frac{m}{3}}}\right) \varphi^{2}\left(e^{-5 \pi \sqrt{\frac{m}{3}}}\right)}{\psi^{2}\left(-e^{-\pi \sqrt{\frac{m}{3}}}\right) \varphi^{2}\left(e^{-\pi \sqrt{\frac{m}{3}}}\right) \psi^{2}\left(-e^{-5 \pi \sqrt{3 m}}\right) \varphi^{2}\left(e^{-5 \pi \sqrt{3 m}}\right)}, \\
V_{m} & :=\frac{G_{\frac{m}{3}}^{2} G_{\frac{25 m}{3}}^{2}}{G_{3 m}^{2} G_{75 m}^{2}}
\end{aligned}
$$


then

$$
\sqrt{A_{m}}+\frac{1}{\sqrt{A_{m}}}=\left(V_{m}+V_{m}^{-1}\right)+1
$$

Proof By Entry 61 of Chap. 25 of Ramanujan's notebooks [5, (61.18), p. 218], we have

$$
P Q+\frac{1}{P Q}=\left(\frac{P}{Q}\right)^{2}+\left(\frac{Q}{P}\right)^{2}+1
$$

where

$$
P=\frac{f\left(-q^{6}\right) f\left(q^{5}\right)}{q^{\frac{1}{4}} f\left(-q^{2}\right) f\left(q^{15}\right)}
$$

and

$$
Q=\frac{f\left(q^{3}\right) f\left(-q^{10}\right)}{q^{\frac{3}{4}} f(q) f\left(-q^{30}\right)} .
$$

Let $q=e^{-\pi \sqrt{\frac{m}{3}}}$. Using Entry 24(iii) of Chap. 16 of Ramanujan's notebooks [4, p. 39] in (3.7), we find that

$$
A_{m}:=\frac{f^{2}\left(q^{3}\right) f^{2}\left(-q^{6}\right) f^{2}\left(q^{5}\right) f^{2}\left(-q^{10}\right)}{q^{2} f^{2}(q) f^{2}\left(-q^{2}\right) f^{2}\left(q^{15}\right) f^{2}\left(-q^{30}\right)} .
$$

Using (3.11) in (3.10), we deduce that

$$
\sqrt{A_{m}}+\frac{1}{\sqrt{A_{m}}}=\frac{G_{\frac{m}{3}}^{2} G_{\frac{25 m}{3}}^{2}}{G_{3 m}^{2} G_{75 m}^{2}}+\frac{G_{3 m}^{2} G_{75 m}^{2}}{G_{\frac{m}{3}}^{2} G_{\frac{25 m}{3}}^{2}}+1 .
$$

Using (3.8) in (3.12), we obtain the required result.

Theorem 3.3 If $A_{m}$ is defined as in (3.7) and

$$
V_{m}:=\frac{G_{3 m}^{3} G_{\frac{25 m}{3}}^{3}}{G_{\frac{m}{3}}^{3} G_{75 m}^{3}}
$$

then

$$
\sqrt{A_{m}}+\frac{1}{\sqrt{A_{m}}}=V_{m}+V_{m}^{-1}-4 \text {. }
$$

Proof By Entry 59 of Chap. 25 of Ramanujan's notebooks [5, (59.9), p. 215], we have

$$
R^{3}+R^{2} Q^{2}=Q^{3}-R Q
$$


where

$$
R=\frac{f\left(q^{3}\right) f\left(q^{5}\right)}{q^{\frac{1}{3}} f(q) f\left(q^{15}\right)}
$$

and

$$
Q=\frac{f\left(-q^{6}\right) f\left(-q^{10}\right)}{q^{\frac{2}{3}} f\left(-q^{2}\right) f\left(-q^{30}\right)} .
$$

Squaring both sides of the identity (3.15), we obtain

$$
R Q+\frac{1}{R Q}=\left(\frac{Q}{R}\right)^{3}+\left(\frac{R}{Q}\right)^{3}-4
$$

Using Entry 24(iii) of Chap. 16 of Ramanujan's notebooks [4, p. 39] in (3.7) with $q=e^{-\pi \sqrt{\frac{m}{3}}}$, we find that

$$
A_{m}=\frac{f^{2}\left(q^{3}\right) f^{2}\left(q^{5}\right) f^{2}\left(-q^{6}\right) f^{2}\left(-q^{10}\right)}{q^{2} f^{2}(q) f^{2}\left(q^{15}\right) f^{2}\left(-q^{2}\right) f^{2}\left(-q^{30}\right)} .
$$

Using (3.17) in (3.16) and then using (3.13) in the resulting identity, we obtain (3.14).

Theorem 3.4 If $m$ is any positive rational,

$$
\begin{aligned}
A_{m} & :=\frac{a_{m, 3}}{a_{49 m, 3}} \\
& =\frac{e^{\pi \sqrt{3 m}} \psi^{2}\left(-e^{-\pi \sqrt{3 m}}\right) \varphi^{2}\left(e^{-\pi \sqrt{3 m}}\right) \psi^{2}\left(-e^{-7 \pi \sqrt{\frac{m}{3}}}\right) \varphi^{2}\left(e^{-7 \pi \sqrt{\frac{m}{3}}}\right)}{\psi^{2}\left(-e^{-\pi \sqrt{\frac{m}{3}}}\right) \varphi^{2}\left(e^{-\pi \sqrt{\frac{m}{3}}}\right) \psi^{2}\left(-e^{-7 \pi \sqrt{3 m}}\right) \varphi^{2}\left(e^{-7 \pi \sqrt{3 m}}\right)} \\
V_{m} & :=\frac{G_{\frac{m}{3}} G_{\frac{49 m}{3}}}{G_{3 m} G_{147 m}}
\end{aligned}
$$

then

$$
\sqrt{A_{m}}-\frac{1}{\sqrt{A_{m}}}=\left(V_{m}-V_{m}^{-1}\right)\left[\left(V_{m}-V_{m}^{-1}\right)-1\right] .
$$

Proof In [8], [9, Theorem 3.1(ii)], we have

$$
R Q-\frac{1}{R Q}=\left(\frac{Q}{R}\right)^{3}-\left(\frac{R}{Q}\right)^{3}+4\left(\frac{R}{Q}-\frac{Q}{R}\right),
$$

where

$$
R=\frac{q^{\frac{1}{2}} f(q) f\left(q^{21}\right)}{f\left(q^{3}\right) f\left(q^{7}\right)}
$$


and

$$
Q=\frac{q f\left(-q^{2}\right) f\left(-q^{42}\right)}{f\left(-q^{6}\right) f\left(-q^{14}\right)} .
$$

Using Entry 24(iii) of Chap. 16 of Ramanujan's notebooks [4, p. 39] in (3.18) with $q=e^{-\pi \sqrt{\frac{m}{3}}}$, we find that

$$
A_{m}=\frac{q^{3} f^{2}\left(q^{3}\right) f^{2}\left(-q^{6}\right) f^{2}\left(q^{7}\right) f^{2}\left(-q^{14}\right)}{f^{2}(q) f^{2}\left(-q^{2}\right) f^{2}\left(q^{21}\right) f^{2}\left(-q^{42}\right)} .
$$

Using (3.22) and (3.19) in (3.21), we obtain the required result (3.20).

Theorem 3.5 If $m$ is any positive rational,

$$
\begin{aligned}
A_{m} & :=a_{m, 3} a_{121 m, 3} \\
& =\frac{9 e^{-2 \pi \sqrt{3 m}} \psi^{2}\left(-e^{-\pi \sqrt{3 m}}\right) \varphi^{2}\left(e^{-\pi \sqrt{3 m}}\right) \psi^{2}\left(-e^{-11 \pi \sqrt{3 m}}\right) \varphi^{2}\left(e^{-11 \pi \sqrt{3 m}}\right)}{\psi^{2}\left(-e^{-\pi \sqrt{\frac{m}{3}}}\right) \varphi^{2}\left(e^{-\pi \sqrt{\frac{m}{3}}}\right) \psi^{2}\left(-e^{-11 \pi \sqrt{\frac{m}{3}}}\right) \varphi^{2}\left(e^{-11 \pi \sqrt{\frac{m}{3}}}\right)}, \\
V_{m} & :=\frac{G_{\frac{m}{3}} G_{\frac{121 m}{3}}}{G_{3 m} G_{363 m}},
\end{aligned}
$$

then

$$
\sqrt{A_{m}}+\frac{1}{\sqrt{A_{m}}}=\frac{1}{3}\left[\left(V_{m}+V_{m}^{-1}\right)\left(\left(V_{m}+V_{m}^{-1}-2\right)^{2}-7\right)-12\right] .
$$

Proof By Theorem 3.1(i) in [9] and [8], we find that

$$
R Q+\frac{9}{R Q}=\left(\frac{Q}{R}\right)^{3}+\left(\frac{R}{Q}\right)^{3}+4\left(\frac{Q}{R}\right)^{2}+4\left(\frac{R}{Q}\right)^{2}-4
$$

where

$$
R=\frac{f(q) f\left(q^{11}\right)}{q f\left(q^{3}\right) f\left(q^{33}\right)}
$$

and

$$
Q=\frac{f\left(-q^{2}\right) f\left(-q^{22}\right)}{q^{2} f\left(-q^{6}\right) f\left(-q^{66}\right)} .
$$

Using Entry 24(iii) of Chap. 16 of Ramanujan's notebooks [4, p. 39] in (3.23) with $q=e^{-\pi \sqrt{\frac{m}{3}}}$, we find that

$$
A_{m}=\frac{9 q^{6} f^{2}\left(q^{3}\right) f^{2}\left(-q^{6}\right) f^{2}\left(q^{33}\right) f^{2}\left(-q^{66}\right)}{f^{2}(q) f^{2}\left(-q^{2}\right) f^{2}\left(q^{11}\right) f^{2}\left(-q^{22}\right)} .
$$

Using (3.27) and (3.24) in (3.26), we obtain (3.25). 
Theorem 3.6 If $m$ is any positive rational and $a_{m, n}$ is defined as in (2.1),

$$
\begin{aligned}
A_{m} & :=\frac{a_{169 m, 3}}{a_{m, 3}}, \\
V_{m} & :=\frac{G_{\frac{169 m}{3}} G_{3 m}}{G_{507 m} G_{\frac{m}{3}}},
\end{aligned}
$$

then

$$
\sqrt{A_{m}}+\frac{1}{\sqrt{A_{m}}}=\left(V_{m}+V_{m}^{-1}\right)\left[\left(V_{m}+V_{m}^{-1}-2\right)^{2}+1\right] .
$$

Proof By Theorem 3.1(iv) in [9], we find that

$$
\begin{aligned}
& R Q+\frac{1}{R Q} \\
& =\left(\frac{Q}{R}\right)^{3}+\left(\frac{R}{Q}\right)^{3}-4\left(\frac{Q}{R}\right)^{2}-4\left(\frac{R}{Q}\right)^{2}+8\left(\frac{Q}{R}\right)+8\left(\frac{R}{Q}\right)-8,
\end{aligned}
$$

where

$$
R=\frac{f\left(q^{3}\right) f\left(q^{13}\right)}{q f(q) f\left(q^{39}\right)}
$$

and

$$
Q=\frac{f\left(-q^{6}\right) f\left(-q^{26}\right)}{q^{2} f\left(-q^{2}\right) f\left(-q^{78}\right)} .
$$

Using Entry 24(iii) of Chap. 16 of Ramanujan's notebooks [4, p. 39] in (3.28) with $q=e^{-\pi \sqrt{\frac{m}{3}}}$, we find that

$$
A_{m}=\frac{q^{6} f^{2}(q) f^{2}\left(-q^{2}\right) f^{2}\left(q^{39}\right) f^{2}\left(-q^{78}\right)}{f^{2}\left(q^{3}\right) f^{2}\left(-q^{6}\right) f^{2}\left(q^{13}\right) f^{2}\left(-q^{26}\right)} .
$$

Using (3.32) and (3.29) in (3.31), we obtain (3.30).

Theorem 3.7 If $m$ is any positive rational and $a_{m, n}$ is defined as in (2.1),

$$
A_{m}:=\frac{a_{49 m, 5}}{a_{m, 5}}
$$

and

$$
V_{m}:=\frac{G_{5 m} G_{\frac{49 m}{5}}}{G_{\frac{m}{5}} G_{245 m}}
$$

then

$$
\sqrt{A_{m}}+\frac{1}{\sqrt{A_{m}}}=\left[\left(V_{m}+V_{m}^{-1}\right)\left[\left(V_{m}+V_{m}^{-1}-2\right)^{2}-9\right]-4\right] .
$$


Proof By using Entry 12(i), (ii) of Chap. 17 of Ramanujan's notebooks [4, p. 124] in Entry 19(iv) of Chap. 20 of Ramanujan's notebooks [4, p. 426], we find that

$$
\begin{aligned}
& R Q+\frac{1}{R Q} \\
& =\left(\frac{Q}{R}\right)^{3}+\left(\frac{R}{Q}\right)^{3}-4\left(\frac{Q}{R}\right)^{2}-4\left(\frac{R}{Q}\right)^{2}+8\left(\frac{Q}{R}\right)+8\left(\frac{R}{Q}\right)-12
\end{aligned}
$$

where

$$
R=\frac{f\left(q^{5}\right) f\left(q^{7}\right)}{q f(q) f\left(q^{35}\right)}
$$

and

$$
Q=\frac{f\left(-q^{10}\right) f\left(-q^{14}\right)}{q^{2} f\left(-q^{2}\right) f\left(-q^{70}\right)} .
$$

Using Entry 24(iii) of Chap. 16 of Ramanujan's notebooks [4, p. 39] in (3.33) with $q=e^{-\pi \sqrt{\frac{m}{5}}}$, we find that

$$
A_{m}=\frac{q^{6} f^{2}(q) f^{2}\left(-q^{2}\right) f^{2}\left(q^{35}\right) f^{2}\left(-q^{70}\right)}{f^{2}\left(q^{5}\right) f^{2}\left(-q^{10}\right) f^{2}\left(q^{7}\right) f^{2}\left(-q^{14}\right)} .
$$

Using (3.37) and (3.34) in (3.36), we obtain the required result.

Theorem 3.8 If $m$ is any positive rational,

$$
\begin{aligned}
& 5\left[\sqrt{a_{m, 5} a_{9 m, 5}}+\frac{1}{\sqrt{a_{m, 5} a_{9 m, 5}}}\right] \\
& \quad=\frac{a_{m, 5}}{a_{9 m, 5}}+\frac{a_{9 m, 5}}{a_{m, 5}}+\left[3 \sqrt{\frac{a_{m, 5}}{a_{9 m, 5}}}+3 \sqrt{\frac{a_{9 m, 5}}{a_{m, 5}}}-6\right] .
\end{aligned}
$$

Proof By Entry 65 of Chap. 25 of Ramanujan's notebooks [5, p. 230], we have

$$
M N+\frac{25}{M N}=\left(\frac{N}{M}\right)^{2}+\left(\frac{M}{N}\right)^{2}+3\left(\frac{N}{M}\right)+3\left(\frac{M}{N}\right)-6
$$

where

$$
M=\frac{f(q) f\left(-q^{2}\right)}{q^{\frac{1}{2}} f\left(q^{5}\right) f\left(-q^{10}\right)}
$$

and

$$
N=\frac{f\left(q^{3}\right) f\left(-q^{6}\right)}{q^{\frac{3}{2}} f\left(q^{15}\right) f\left(-q^{30}\right)} .
$$


Using (2.1) with $q=e^{-\pi \sqrt{\frac{m}{5}}}$, we find that

$$
M=\frac{\sqrt{5}}{\sqrt{a_{m, 5}}} \quad \text { and } \quad N=\frac{\sqrt{5}}{\sqrt{a_{9 m, 5}}} .
$$

Using (3.40) in (3.39), we obtain (3.38).

Corollary 3.1 Putting $m=1$ in (3.38) and then solving the equation, we obtain

$$
a_{9,5}=(2-\sqrt{3})^{2} .
$$

Acknowledgements The authors would like to thank Prof. B.C. Berndt for providing a reprint [7] and his encouragement. The authors are also thankful to Prof.C. Adiga for his useful suggestions during the preparation of the paper. The authors are grateful to the referee for his useful comments which considerably improves the quality of the paper.

\section{References}

1. Adiga, C., Berndt, B.C., Bhargava, S., Watson, G.N.: Chapter 16 of Ramanujan's second notebook: Theta-function and $q$-series. Mem. Am. Math. Soc. 53(315) (1985)

2. Adiga, C., Mahadeva Naika, M.S., Shivashankara, K.: On some $P-Q$ eta-function identities of Ramanujan. Indian J. Math. 44(3), 253-267 (2002)

3. Adiga, C., Kim, T., Mahadeva Naika, M.S., Madhusudhan, H.S.: On Ramanujan's cubic continued fraction and explicit evaluations of theta-function. Indian J. Pure Appl. Math. 35(9), 1047-1062 (2004)

4. Berndt, B.C.: Ramanujan's Notebooks, Part III. Springer, New York (1991)

5. Berndt, B.C.: Ramanujan's Notebooks, Part IV. Springer, New York (1994)

6. Berndt, B.C.: Ramanujan's Notebooks, Part V. Springer, New York (1998)

7. Berndt, B.C., Chan, H.H., Zhang, L.-C.: Ramanujan's remarkable product of the theta-functions. Proc. Edinburgh Math. Soc. 40, 583-612 (1997)

8. Bhargava, S., Adiga, C., Mahadeva Naika, M.S.: A new class of modular equations akin to Ramanujan's $P-Q$ eta-function identities and some evaluations therefrom. Adv. Stud. Contemp. Math. 5(1), 37-48 (2002)

9. Bhargava, S., Adiga, C., Mahadeva Naika, M.S.: A new class of modular equation in Ramanujan's alternative theory of elliptic function of signature 4 and some new $P-Q$ eta-function identities. Indian J. Math. 45(1), 23-39 (2003)

10. Borwein, J.M., Borwein, P.B.: Pi and AGM. Wiley, New York (1987)

11. Mahadeva Naika, M.S.: $P-Q$ eta-function identities and computations of Ramanujan-Weber class invariants. J. Indian Math. Soc. 70(1-4), 121-134 (2003)

12. Ramanujan, S.: Notebooks (2 volumes). Tata Institute of Fundamental Research, Bombay (1957) 